\title{
Omentin-1 in serum and seminal plasma correlate with semen quality
}

\section{Original Article}

\author{
Sahar A. Ismail', Ali M. Mahran', Eman Mosaad', Suzan Kamel-ElSayed ${ }^{3}$ \\ ${ }^{1}$ Department of Dermatology, Venereology and Andrology, Faculty of Medicine, ${ }^{2}$ Department \\ of Clinical Pathology, South Egypt Cancer Institute, Assiut University, Egypt, ${ }^{3}$ Department of \\ Foundational Medical Sciences, William Beaumont School of Medicine, Oakland University \\ MI USA.
}

\begin{abstract}
Introduction: Adipokines are differently regulated in the male reproductive tract and they were reported to affect sperm functionality. Serum omentin-1 was reported to have a range of effects on various cell types.

Aim: To explore the presence of omentin-1 in human semen, to investigate its relation with semen parameters in both fertile and infertile men and to compare its serum and seminal plasma levels in particular conditions such as; smoking, varicocele and leukocytospermia.

Methods: Thirty nine infertile and 26 fertile men were enrolled in this case control study. They were subjected to history taking, clinical/physical examination, semen analysis, and measurement of omentin-1 in serum and seminal plasma, DNA fragmentation index (DFI) and seminal reactive oxygen species (ROS).

Results: Omentin-1 was detected in all analyzed semen and serum samples $(n=65)$. The concentration of omentin-1 was significantly higher in seminal plasma than in serum. Serum and seminal omentin-1 were positively correlated with sperm concentration and percentage of progressive motility and were negatively correlated with percentage of abnormal forms, DFI and ROS. The mean seminal plasma level of omentin-1 was significantly decreased in smokers and in patients with leukocytospermia. The mean serum level of omentin-1 was significantly decreased in patients with varicocele. Both serum and seminal omentin-1 levels were significantly lower in infertile patients than fertile men.

Conclusions: Semen and serum Omentin-1 correlate with semen quality. In cases of leukocytospermia and smoking, seminal omentin-1 concentrations were decreased, suggesting that it may play a regulatory role in inflammation of the male reproductive system.
\end{abstract}

Key Words: Omentin-1; semen parameters; infertility; varicocele; leukocytospermia

Received: 28 August 2017 , Accepted: 17 September 2017

Corresponding Author: Sahar A. Ismail, Tel.: +201008899446, Fax: 0020-88-2333327 E-mail: saharsotohy@yahoo.com

ISSN: 2090-6048, September 2017, Vol. 7, No. 4

\section{INTRODUCTION}

Omentin-1, (intelectin-1, and endothelial lectin HL-1 $)^{[1]}$ is an adipose hormone that is expressed in adipose tissue, placenta and ovary ${ }^{[2]}$. Current available data suggested its range of effects on various cell types ${ }^{[3-4]}$. Serum omentin-1 concentrations were reported to correlate passively with BMI and waist circumference ${ }^{[5]}$. Decreased serum level of omentin-1 was reported in polycystic ovary syndrome $^{[6]}$, diabetes ${ }^{[7]}$ and coronary artery disease ${ }^{[8]}$. Moreover, omentin was reported to prevent apoptosis of cardiomyoblasts via inhibition of mitochondrial ROS production $^{[9]}$.

There is limited data about different adipokines and their role in male reproductive system ${ }^{[10-12]}$. Thomas and colleagues $^{[12]}$ studied different adipokines concentrations in human serum and seminal plasma and reported that adipokines are differently regulated in the male, reproductive tract in comparison to peripheral blood, and they could affect sperm functionality.

There is currently no available data on the potential role of omentin-1 in male infertility. The aims of this study were to explore the presence of omentin-1in human semen, to investigate its correlation with different semen parameters in both fertile and infertile men and to compare its serum and seminal plasma levels in some conditions affecting semen quality such as; smoking, varicocele and leukocytospermia.

\section{PATIENTS AND METHODS}

Patients have been recruited from those attending the Andrology outpatient clinic, Assiut University Hospitals for infertility problem (from July 2016 through September 
2016). Thirty nine patients were enrolled for this study according to the inclusion criteria; non-azoospermic men who did not achieve pregnancy after 2 years of unprotected sexual intercourse with normal fertile wife. The patients showed normal concentrations of FSH, LH, and $\mathrm{T}$ evaluated in serum (Roche, Cobas e411 analyzer). Patients with history of diabetes mellitus, chemotherapy, radiotherapy, chronic illness, or medication were excluded from the study.

Twenty six volunteer men who have a pregnant wife (more than 12 weeks of gestation) or have a baby less than 3 months old were recruited from the ante-natal care, vaccination clinics attendee, and the employees of Assiut University Hospital to be included as fertile control. They had time to pregnancy (TTP) less than one year. All subjects provided informed written consent before the inclusion in this study, which was approved by the Ethics Committee of School of Medicine Assiut University.

Evaluation of the participants included medical history, clinical and physical examinations. In addition, scrotal color duplex Doppler ultrasonography was used to explore the possible presence of varicocele. Semen samples were obtained from both fertile and infertile men. Weight, height and waist circumference of all participants were measured. The body mass index (BMI) was calculated as weight height $2\left(\mathrm{~kg} / \mathrm{m}^{2}\right)$ and subjects were classified into: normal weight (BMI 18-25 kg/ m²), overweight (BMI $>25-30 \mathrm{~kg} /$ $\left.\mathrm{m}^{2}\right)$ or obese $\left(\mathrm{BMI}>30 \mathrm{~kg} / \mathrm{m}^{2}\right)$. Waist circumference was measured at the mid-point between the lower borders of the rib cage and the iliac crest.

\section{Semen Analysis:}

Standard semen analysis was performed according to the guidelines of the WHO manual for examination of human semen. ${ }^{[13]}$ Participants were asked to provide a semen sample (via masturbation) into a sterile plastic container after sexual abstinence of 2 -7 days. The samples were left to liquefy in a $37^{\circ} \mathrm{C}$ incubator, and were analyzed within one hour of delivery. Standard procedures included macroscopic parameters (semen volume, viscosity and $\mathrm{pH}$ ) and microscopic parameters (sperm concentration, motility, viability, morphology and seminal leukocytic count). Hypo-osmotic swelling test was used for assessment of sperm viability, Spermac staining was used for preparation of morphology slides. ${ }^{[14]}$ In addition, peroxidase-staining test was used for seminal leukocyte quantification. A concentration of $>1 \times 10^{6}$ cells $/ \mathrm{mL}$ was identified as leukocytospermia.

All samples were examined by the same investigator and results were verified by a second observer. Two aliquots of thoroughly mixed semen samples were taken for replicate analysis. For each sample, replicate values were compared to check if they were acceptably close
( $<10 \%$ difference). If the difference was high, the assessment was repeated on two new semen sample preparations.

\section{Omentin- 1 Measurements:}

Omentin -1was assayed in semen and serum samples of 65 individuals including patients and control. One hour after collection, $500 \mu 1$ of the whole semen were recovered from each sample and centrifuged (1500 rpm for 15 minutes). The supernatant (seminal plasma) was collected and immediately stored at $-80^{\circ} \mathrm{C}$ until time of analysis.

Blood samples were collected in the morning (9:00 - 10:30 AM) after 12 hours of fasting. They were drawn in color-coded vacutainer tubes (BD). Blood was left to clot for approximately 60 minutes, centrifuged at $4{ }^{\circ} \mathrm{C}$, and stored at $-80^{\circ} \mathrm{C}$ until analysis was performed. Omentin -1level was determined by ELISA using the Omentin (Human) ELISA kit (SinoGene Clone Biotech Co.,Ltd). Following the manufacturer's instructions, the results were expressed in $\mathrm{ng} / \mathrm{mL}$.

\section{Sperm DNA fragmentation index assessment (DFI):}

DFI was performed using flowcytometry (DAKOCytomation, Glostrup, Denmark) by Coulter (Beckman Coulter, Fullerton, CA) based on the fluorescence emission from sperms stained with propidium iodide (PI) and excitation with a 488-nanometer (nm) argon laser ${ }^{[15]}$. Fresh semen samples were diluted with phosphate-buffered saline (PBS) to $2 \times 10^{6} \mathrm{sperm} / \mathrm{mL}$. Fifty $\mu \mathrm{L}$ were incubated with $100 \mu \mathrm{L}$ lysing reagent and kept for 15 seconds, then $2 \mathrm{~mL}$ of PI was added and mixed with tube. Immediately after staining, tube acquisition was performed by flowcytometry. The intensity of the emission corresponds to the DNA content. Flowcytometric analysis displays a constant and characteristic bimodal nonartifactual DNA pattern indicating the presence of two different populations. The main population was represented by a peak followed by a shoulder ("marginal population"), which represents a sperm group altered in the nuclear condensation, yielding unstable chromatin which appears more stainable with PI. The percentage of sperm cells with DNA damage was automatically calculated after acquisition of 5000 sperms representing DFI.

\section{Seminal Reactive oxygen species measurement (ROS):}

They were measured in fresh semen samples by assessing the chemiluminescence activity using luminol (5-amino-2,3 dihydro-1,4 phtalazindione reagent) (MP Biomedicals, Irvine, CA $)^{[16]}$. Liquefied semen specimens were centrifuged at $300 \mathrm{~g}$ for 7 minutes and seminal plasma was removed. The pellet was washed twice in PBS by centrifugation at $300 \mathrm{~g}$ for 5 minutes and then re-suspended 
in PBS at a concentration of $20 \times 10^{6}$ sperm $/ \mathrm{mL}$. Ten $\mathrm{mL}$ luminol, (as a probe) was added to the aliquot. Levels of Seminal plasma ROS were determined by assessing chemiluminescence activity by Autolamat luminometer (Berthold Technologies, Bad Wildbad, Germany) in the integrated mode for 15 minutes. Results were presented as Relative light Unit (RLU) per 20 million sperm.

\section{Statistical Analysis:}

Statistical analysis of the data was done using SPSS version 21 program (SPSS Inc., Chicago, IL, USA). Data were expressed as mean and SD, t-test was used in comparisons of numerical parametric data and MannWhitney $U$ test for non parametric data. The Pearson correlation coefficient was applied for bivariate correlation analysis. $P$ value of $<0.05$ was considered significant.

\section{RESULTS}

Sixty five participants were enrolled in the study; 39 of them were infertile patients and 26 were healthy fertile controls. Their mean age was $32.95 \pm 7.87$.

\section{Omentin-1 level in serum and seminal plasma:}

Omentin-1 was detected in all analyzed semen and serum samples $(n=65)$. The concentration of omentin-1 was significantly higher $(P<0.05)$ in seminal plasma $(56.93 \pm 12.43)$ than in serum $(46.55 \pm 12.72)$ and it was correlated significantly $(\mathrm{r}=0.69, P<0.001)$ between both sample matrices.

\section{Omentin-1 levels in obese men:}

The normal weight men $(\mathrm{n}=31$, mean age 31.65 $\pm 6.17)$ had statistically significant $(P<0.05)$ higher serum omentin-1 level $(51.77 \pm 12.97)$ compared with the overweight/obese men ( $\mathrm{n}=34$, mean age $34.15 \pm 9.07)$ $(41.79 \pm 10.59)$. In addition, level of omentin-1 in seminal plasma was insignificantly higher in normal weight men $(60.09 \pm 12.89)$ compared with the overweight/obese group $(54.06 \pm 11.44)$. In contrast, both BMI and waist circumference had a statistically significant negative correlation with the levels of omentin-1 in serum ( $\mathrm{r}=$ -0.38, $P<0.01$ and $\mathrm{r}=-0.53, P<0.001$ respectively) and seminal plasma $(\mathrm{r}=-0.25, P<0.05$ and $\mathrm{r}=-0.39$, $P<0.001$, respectively).

\section{Omentin-1 levels and semen parameters:}

Serum level of omentin- 1 in all participants was correlated positively with sperm concentration $(\mathrm{r}=0.31$, $P<0.05)$ and percentage of progressive motility $(\mathrm{r}=0.35$, $P<0.01)$; and negatively with percentage of abnormal forms ( $\mathrm{r}=-0.42, P<0.001)$, DFI $(\mathrm{r}=-0.43, P<0.001)$ and ROS $(\mathrm{r}=-0.27, P<0.001)$. Likewise, the seminal plasma omentin-1 level correlated positively with sperm concentration $(\mathrm{r}=0.40, P<0.001)$ and percentage of progressive motility $(\mathrm{r}=0.57, P<0.001)$; and negatively with percentage of abnormal forms $(\mathrm{r}=-0.59, P<0.001)$, DFI $(\mathrm{r}=-0.49, P<0.001)$ and ROS $(\mathrm{r}=-0.42, P<0.001)$.

\section{Effect of smoking, varicocele and} leukocytospermia on omentin-1 levels (Table 1):

The mean seminal plasma level of omentin-1 was significantly lower $(P<0.05)$ in smokers $(54.15 \pm 13.83)$ compared with nonsmokers $(61.12 \pm 10.8)$ and in patients with leukocytospermia $(51.0 \pm 9.50)$ compared with those without leukocytospermia $(59.04 \pm 12.75)(P<0.05)$. The mean serum level of omentin-1 was significantly lower $(P<0.05)$ in patients with varicocele $(41.4 \pm 9.68)$ compared with those without varicocele (48.84 \pm 13.33$)$.

\section{Omentin-1 levels in fertile versus infertile subjects (Table 2):}

Comparing the two main groups of the study (39 infertile patients and 26 age and BMI matched fertile control) revealed that semen parameters, DFI and seminal ROS were significantly altered $(P<0.001)$. The mean seminal plasma level of omentin-1 was significantly lower $(P<0.001)$ in infertile patients $(49.95$ $\pm 9.07)$ compared with fertile control $(67.42 \pm 8.97)$ and the mean serum level of omentin-1 was also significantly lower $(P<0.001)$ in infertile patients $(40.67 \pm 11.24)$ compared with fertile control $(55.38 \pm 9.36)$. 
Table 1: Different variables reported in smokers vs. nonsmokers, varicocele vs. no varicocele and leukocytospermia vs. no leukocytospermia

\begin{tabular}{|c|c|c|c|c|c|c|}
\hline & \multirow{2}{*}{$\begin{array}{c}\text { Smoking Yes } \\
\qquad(\mathrm{n}=39) \\
\text { Yes }(n=39)\end{array}$} & \multicolumn{3}{|c|}{ Varicocele } & \multicolumn{2}{|c|}{ Leukocytospermia } \\
\hline & & No $(n=26)$ & Yes $(n=20)$ & No $(n=45)$ & Yes $(n=17)$ & No $(n=48)$ \\
\hline Age (year) & $31.81 \pm 6.9$ & $33.72 \pm 8.4$ & $32.35 \pm 7.7$ & $33.22 \pm 7.9$ & $30.06 \pm 8.3$ & $31.15 \pm 6.9$ \\
\hline $\mathrm{BMI}(\mathrm{kg} / \mathrm{m} 2)$ & $25.59 \pm 4.0$ & $26.05 \pm 4.9$ & $27.2 \pm 3.4$ & $25.14 \pm 4.7$ & $25.22 \pm 4.4$ & $25.97 \pm 4.4$ \\
\hline Semen volume $(\mathrm{ml})$ & $3.60 \pm 1.1$ & $3.34 \pm 1.7$ & $3.05 \pm 1.4$ & $3.62 \pm 1.5$ & $3.16 \pm 1.2$ & $3.54 \pm 1.6$ \\
\hline $\begin{array}{l}\text { Sperm count (106/ } \\
\text { mL) }\end{array}$ & $23.16 \pm 22.8$ & $68.92 \pm 31.3 * *$ & $27.87 \pm 22.9$ & $47.51 \pm 37.4 * *$ & $36.39 \pm 33.8$ & $43.26 \pm 35.2$ \\
\hline $\begin{array}{l}\text { Progressive } \\
\text { motility (\%) }\end{array}$ & $29.23 \pm 17.5$ & $41.42 \pm 19.2^{*}$ & $33.85 \pm 15.0$ & $35.33 \pm 20.7$ & $24.24 \pm 17.0$ & $37.60 \pm 18.6^{* *}$ \\
\hline $\begin{array}{l}\text { Abnormal forms } \\
(\%)\end{array}$ & $71.41 \pm 10.1$ & $64.23 \pm 11.8^{*}$ & $72.75 \pm 9.3$ & $66.67 \pm 11.6^{*}$ & $75.29 \pm 11.1$ & $66.15 \pm 10.4^{*}$ \\
\hline DFI (\%) & $36.02 \pm 9.3$ & $29.0 \pm 9.4^{*}$ & $34.75 \pm 5.4$ & $28.84 \pm 10.7 *$ & $34.76 \pm 8.3$ & $31.83 \pm 7.8$ \\
\hline ROS (RLU) & 125.99 & $24.64 * *$ & 93.55 & $36.18 *$ & 325.35 & $19.13 * * *$ \\
\hline $\begin{array}{l}\text { Omentin-1 semen } \\
(\mathrm{ng} / \mathrm{ml})\end{array}$ & $54.15 \pm 12.1$ & $61.12 \pm 11.9^{*}$ & $52.95 \pm 9.9$ & $58.71 \pm 13.1$ & $51.0 \pm 9.5$ & $59.04 \pm 12.8 *$ \\
\hline $\begin{array}{l}\text { Omentin-1 serum } \\
(\mathrm{ng} / \mathrm{ml})\end{array}$ & $45.18 \pm 13.8$ & $48.62 \pm 10.8$ & $41.4 \pm 9.7$ & $48.84 \pm 13.3 *$ & $44.59 \pm 12.2$ & $47.25 \pm 12.9$ \\
\hline
\end{tabular}

Smoking: $>10$ cigarette per day for more than 5 years; Leukocytospermia: $\left(>1 \times 10^{6}\right.$ leukocytes $/ \mathrm{mL}$ of semen)

Table 2: Comparison between the considered variables in infertile versus fertile subjects

\begin{tabular}{|c|c|c|}
\hline & $\begin{array}{l}\text { Infertile subjects } \\
\qquad(\mathrm{n}=39)\end{array}$ & $\begin{array}{l}\text { Fertile subjects } \\
\qquad(\mathrm{n}=26)\end{array}$ \\
\hline Age (year) & $34.41 \pm 9.3$ & $30.77 \pm 4.5$ \\
\hline BMI (kg/m2) & $25.92 \pm 4.3$ & $25.56 \pm 4.7$ \\
\hline Waist circumference $(\mathrm{cm})$ & $96.41 \pm 9.9$ & $94.50 \pm 10.1$ \\
\hline Semen volume (ml) & $3.23 \pm 1.6$ & $3.76 \pm 1.3$ \\
\hline Sperm count $(106 / \mathrm{mL})$ & $28.24 \pm 27.5$ & $61.31 \pm 35.4^{*}$ \\
\hline Progressive motility (\%) & $22.23 \pm 14.7$ & $51.92 \pm 7.0 *$ \\
\hline Abnormal forms (\%) & $75.00 \pm 9.3$ & $58.85 \pm 5.5^{*}$ \\
\hline DFI (\%) & $38.8 \pm 9.3$ & $28.85 \pm 5.7 *$ \\
\hline ROS (RLU) & 270.65 & $12.93 *$ \\
\hline Omentin-1 semen $(\mathrm{ng} / \mathrm{ml})$ & $49.95 \pm 9.07$ & $67.42 \pm 8.97 *$ \\
\hline Omentin-1 serum (ng/ml) & $40.67 \pm 11.24$ & $55.39 \pm 9.36^{*}$ \\
\hline
\end{tabular}

BMI: Body mass index; DFI: DNA fragmentation index; ROS: reactive oxygen species; RLU: relative light unit

Note: ROS values were described as medians and other values were described as mean \pm standard deviation. *: $P$ value $<0.001$ 


\section{DISCUSSION}

We revealed for the first time that omentin-1 can be detected in human serum and in higher concentration in seminal plasma. The reported high levels of omentin-1 in seminal plasma points to special physiologic functions of omentin-1 in this body fluid although its source remains largely uncertain. The circulating blood levels of omentin-1 were significantly correlated with the seminal plasma values. This correlation could be explained by an exchange between these two compartments by a gap via the blood-testicular barrier. While the vascular barrier reduces the entry of serum proteins, omentin- 1 could be taken up into the testes by leakage rather than transport as reported for leptin $^{[17]}$. Otherwise, omentin-1 could be secreted by epididymal adipocytes, assuming a paralleled secretion mechanism similar to that described for omentin-1 produced in subcutaneous or visceral adipose tissue. This hypothesis is supported by the mouse model, in which visceral and epididymal fat increased in parallel with obesity ${ }^{[18]}$. However the presence of paralleled omentin- 1 concentrations in serum and seminal plasma is not a sufficient proof for any secretion or vital action of this adipokine on spermatozoa.

A link between obesity and male infertility in the form of impaired semen quality has been previously reported $^{[12]}$. However the pathogenesis of this acknowledged interaction remains unclear. We found high levels of both serum and seminal omentin- in normal weight compared with overweight/obese men. Moreover, in agreement with previous literature ${ }^{[5]}$, BMI and waist circumference showed inverse correlation with both serum and seminal omentin-1. Thus, impaired omentin-1 in overweight/obese men could represent the pathophysiological link between obesity and male infertility.

Moreover, we also demonstrated that semen and serum levels of omentin-1 were positively correlated to sperm concentration and progressive motility percentage and negatively correlated to abnormal forms percentage, DFI and seminal ROS. Recent studies have reported elevated TNF- $\alpha$ in seminal plasma to be associated with decreased sperm count, motility and normal forms. 19, 20 In addition, multiple studies have demonstrated that excessive levels of ROS can damage normal spermatozoa by inducing lipid peroxidation and DNA damage and are often accompanied with low sperm functions and subfertility ${ }^{[21,22]}$. The reported anti-inflammatory properties of omentin-1, its ability to decrease TNF $\alpha$ induced inflammation in endothelial cells and in vascular smooth muscle cells ${ }^{[23-25]}$ and its ability to prevent apoptosis of cardiomyoblasts through reduction of mitochondrial ROS production 9 may explain its supportive protecting role and introduce it as a potential pharmaco-therapeautic target against male infertility.

We divided the patients into sub groups according to the presence of certain conditions that may affect the semen quality: smoking, varicocele and leukocytospermia. We observed a significant lower level of omentin-1 in semen samples of cigarette smokers compared with nonsmokers concomitant with significant lower sperm count and progressive motility percentage and significant higher DFI and seminal ROS level. These findings are supported by the current dogma that the persistent oxidative burden caused by smoking can generate secondary mediators which may induce inflammation ${ }^{[26]}$.

The presence of leukocytospermia in our patients was concomitant with a reduction of sperm motility and increase percentages of abnormal sperms. Previous studies reported that leukocytospermia could exert a negative effect on semen quality ${ }^{[11,27]}$. We demonstrated that human semen samples with leukocytospermia showed lower levels of omentin-1, concomitant with higher level of seminal ROS, which has been reported to exert a negative effect on sperm quality ${ }^{[21,22]}, \mathrm{We}$ hypothesize that the presence of leukocytes in semen, low level of omentin-1 and decreasing semen quality, could indicate a typical inflammation profile.

In the group of individuals with varicocele the sperm count, was significantly lower than that in individuals without varicocele. Concomitantly, the percentage of abnormal forms, DFI and seminal ROS level were higher in the varicocele group. Such observations confirm the detrimental role of varicocele in spermatogenesis ${ }^{[11,15]}$ It is notable that varicocele in our patients was associated with significant lower level of serum omentin-1and slight lower level of seminal omentin-1 compared with patients without varicocele. These findings present another mechanism by which varicocele impair spermatogenesis, however, this postulate needs further research that is beyond the scope of this study.

It is noteworthy that both serum and semen levels of omentin-1 were significantly lower in infertile patients compared with those observed in control fertile subjects. These results could be of particular interest and deserve attention in the field of male infertility.

\section{CONCLUSION}

We have shown for the first time that omentin -1 in serum and semen is correlated with semen quality. A case can be made that omentin-1 is an important regulator of reproductive events as it can reduce the consequential effect of inflammation on male reproduction, particularly seminal oxidative stress and sperm DNA damage which 
will not only decrease male reproductive failure but may also improve success rates of assisted reproductive technology.

\section{CONFLICT OF INTEREST}

There are no conflicts of interest

\section{REFERENCES}

1. Yang RZ, Lee MJ, Hu H, Pray J, Wu HB, Hansen $\mathrm{BC}$, et al. Identification of omentin as a novel depot-specific adipokine in human adipose tissue: possible role in modulating insulin action. Am J Physiol Endocrinol Metab 2006; 290(6): E1253-1261.

2. Schaffler A, Neumeier M, Herfarth H, Furst A, Scholmerich J, Buchler C. Genomic structure of human omentin, a new adipocytokine expressed in omental adipose tissue. Biochim Biophys Acta 2005;1732:96-102.

3. Suzuki YA, Shin K, Lonnerdal B. Molecular cloning and functional expression of a human intestinal lactoferrin receptor. Biochemistry 2001;40:15771-15779.

4. Maruyama S, Shibata R, Kikuchi R, Izumiya Y, Rokutanda $\mathrm{T}$, Araki S, et al. Fat-derived factor omentin stimulates endothelial cell function and ischemia-induced revascularization via endothelial nitric oxide synthase-dependent mechanism. J Biol Chem 2012;287:408-417.

5. De Souza Batista CM, Yang RZ, Lee MJ, Glynn NM, Yu DZ, Pray J, et al. Omentin plasma levels and gene expression are decreased in obesity. Diabetes, 2007; 56:1655-1661.

6. Mahde A, Shaker M, Al-Mashhadani Z. Study of Omentin 1 and Other Adipokines and Hormones in PCOS Patients. Oman Med J, 2009; 24:108-118.

7. Yan P, Liu D, Long M, Ren Y, Pang J, Li R. Changes of serum omentin levels and relationship between omentin and adiponectin concentrations in type 2 diabetes mellitus. Exp Clin Endocrinol Diabetes, 2011; 119: 257-263.

8. Zhong $\mathrm{X}$, Zhang HY, Tan H, Zhou Y, Liu FL, Chen FQ, Shang DY. Association of serum omentin-1 levels with coronary artery disease. Acta Pharmacol Sin, 2011; 32: 873-878.

9. Kazama K, Okada M, Yamawaki H.Adipocytokine, omentin inhibits doxorubicin-induced H9c2 cardiomyoblasts apoptosis through the inhibition of mitochondrial reactive oxygen species. Biochem Biophys Res Commun. 2015;457(4):602-607.

10. Dupont J, Pollet-Villard X, Reverchon M, Mellouk N, Levy R. Adipokines in human reproduction. Horm Mol Biol Clin Investig. 2015;24(1):11-24.

11. Moretti E, Collodel G, Mazzi L, Campagna M, Iacoponi F, Figura N. Resistin, interleukin-6, tumor necrosis factor-alpha, and human semen parameters in the presence of leukocytospermia, smoking habit, and varicocele. Fertil Steril. 2014;102(2):354-360.

12. Thomas S, Kratzsch D, Schaab M, Scholz M, Grunewald S, Thiery J, et al. Seminal plasma adipokine levels are correlated with functional characteristics of spermatozoa. Fertil Steril. 2013;99(5):1256-1263.

13. World Health Organization. Laboratory Manual for the Examination of Human Semen and SemenCervical Mucus Interaction, 5th ed. Cambridge: Cambridge University Press; 2010.

14. Chan PJ, Corselli JU, Jacobson JD, Patton WC, King A. Spermac stain analysis of human sperm acrosomes. Fertil Steril. 1999;72(1):124-128.

15. Sadek A, Almohamdy AS, Zaki A, Aref M, Ibrahim SM, Mostafa T. Sperm chromatin condensation in infertile men with varicocele before and after surgical repair. Fertil Steril. 2011;95(5): 1705-1708.

16. Wang X, Sharma RK, Gupta A, George V, Thomas AJ, Falcone T, Agarwal A. Alterations in mitochondria membrane potential and oxidative stress in infertile men: a prospective observational study. Fertil Steril. 2003;80(Suppl 2):844-850.

17. Banks WA, McLay RN, Kastin AJ, Sarmiento U, Scully S. Passage of leptin across the blood-testis barrier. Am J Physiol 1999;276:E1099-1104.

18. Soret MG, Kupiecki FP, Wyse BM. Epididymal fat pad alterations in mice with spontaneous obesity and diabetes and with chemically induced obesity. Diabetologia 1974;10(Suppl):639-648.

19. Sanocka D, Jedrzejczak P, Szumała-Kaekol A, Fraczek M, Kurpisz M. Male genital tract inflammation: the role of selected interleukins in regulation of pro-oxidant and antioxidant enzymatic substances in seminal plasma. J Androl. 2003;24(3):448-455. 
20. Azenabor A, Ekun AO, Akinloye O. Impact of Inflammation on Male Reproductive Tract. Journal of Reproduction \& Infertility. 2015;16(3): 123-129.

21. Agarwal A, Saleh RA, Bedaiwy MA. Role of reactive oxygen species in the pathophysiology of human reproduction. Fertil Steril 2003;79:829 843.

22. Agarwal A, Sharma RK, Sharma R, Assidi $\mathrm{M}$, Abuzenadah AM, Alshahrani $\mathrm{S}$, et al. Characterizing semen parameters and their association with reactive oxygen species in infertile men. Reprod Biol Endocrinol. 2014;12:33.

23. Tan BK, Adya R, Randeva HS. Omentin: a novel link between inflammation, diabesity, and cardiovascular disease. Trends Cardiovasc Med. 2010;20(5):143-148.

24. Yamawaki H, Kuramoto J, Kameshima S, Usui T, Okada M, Hara Y. Omentin, a novel adipocytokine inhibits TNF-induced vascular inflammation in human endothelial cells. Biochem Biophys Res Commun. 2011;408(2):339-343.

25. Kazama K, Usui T, Okada M, Hara Y, Yamawaki H. Omentin plays an anti-inflammatory role through inhibition of TNF- $\alpha$-induced superoxide production in vascular smooth muscle cells. Eur $\mathbf{J}$ Pharmacol. 2012;686(1 -3):116-123.

26. Taha EA, Ez-Aldin AM, Sayed SK, Ghandour NM, Mostafa T. Effect of smoking on sperm vitality, DNA integrity, seminal oxidative stress, zinc in fertile men. Urology. 2012;80(4):822-825.

27. Aghazarian A, Stancik I, Pflüge H, Lackner $\mathrm{J}$. Influence of pathogens and moderate leukocytes on seminal interleukin (IL)-6, IL-8, and sperm parameters. Int Urol Nephrol. 2013; 45(2):359-365. 